\title{
THE TESTING AND FUTURE DEVELOPMENTS OF A NERVE STIMULATION DEVICE FOR THE TREATMENT OF MIGRAINES
}

\author{
A Thesis \\ presented to \\ the Faculty of California Polytechnic State University, \\ San Luis Obispo
}

\author{
In Partial Fulfillment \\ of the Requirements for the Degree
}

Master of Science in Engineering with Specialization in Biomedical Engineering

by

Alyssa Hopkins

February 2011 
(C) 2011

Alyssa Hopkins

ALL RIGHTS RESERVED 
COMMITTEE MEMBERSHIP

TITLE:

The Testing and Future Developments of a Nerve Stimulations Device for the Treatment of Migraines

AUTHOR:

Alyssa Erin Hopkins

DATE SUBMITTED:

February 2011

COMMITTEE CHAIR:

Robert Crockett, Professor

COMMITTEE MEMBER: Daniel Walsh, Professor

COMMITTEE MEMBER: Lanny Griffin, Professor 


\begin{abstract}
The Testing and Future Developments of a Nerve Stimulations Device for the Treatment of Migraines

Alyssa Erin Hopkins

The development of neural stimulations devices may help treat many patients who suffer from migraines and chronic headaches via an electrical stimulation to the patient's nerve. The electrical stimulation will mask the pain associated with these diseases. The system is subcutaneously implanted into the back of the neck and recharged via inductive coupling. Discussed is the transmission of signals in nerve fibers and the testing involved with neural stimulation implantable devices. Research was performed for preliminary testing on the charging rates, tests to analyze the heat dissipated during recharging, ergonomic factors to be considered, developing a model to simulate an in vivo environment.
\end{abstract}

Keywords: Neural stimulation, chronic headaches, inductive coupling. 


\section{TABLE OF CONTENTS}

\section{Page}

LIST OF FIGURES

vi

\section{CHAPTER}

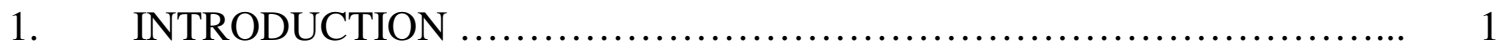

2. DEVICE APPLICATIONS ......................................... 2

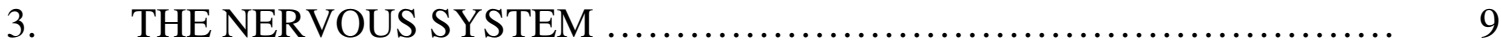

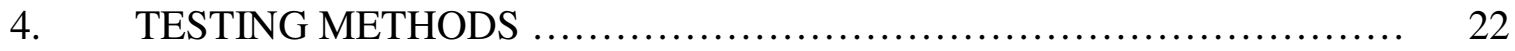

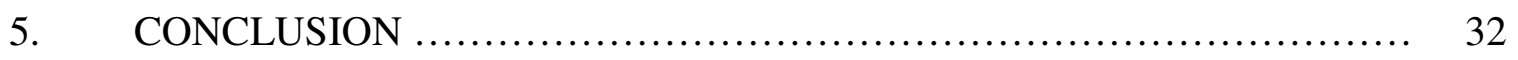

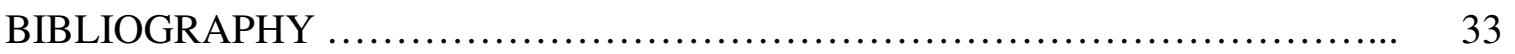




\section{LIST OF FIGURES}

Figure $\quad$ Page

1. Illustration of possible Multi-Electrode bion (MEb) .................... 3

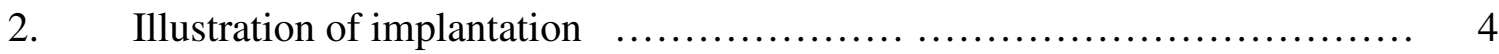

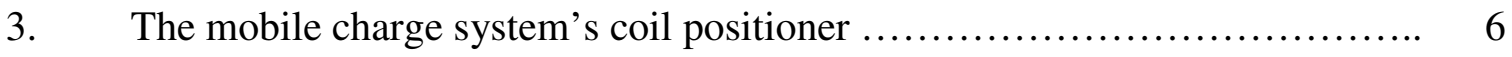

4. Illustration of possible Remote Control ............................... 7

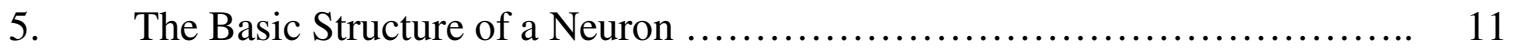

6. The Charge Separation of Ions on a Cell Membrane ..................... 12

7. Voltage Gated Ion Channels and an ATP Sodium-Potassium pump,

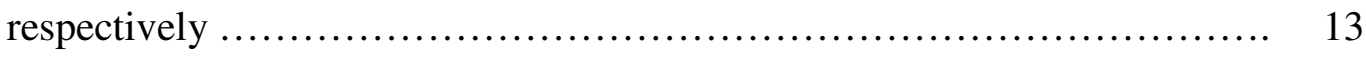

8. A Voltmeter Reading the Membrane Potential of a Neuron ................. 17

9. The stages of an Action Potential ................................... 19

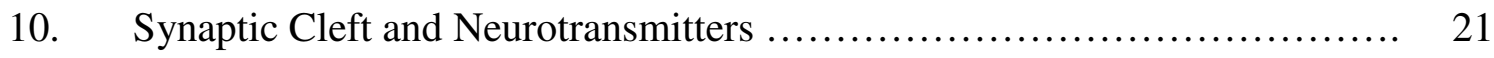

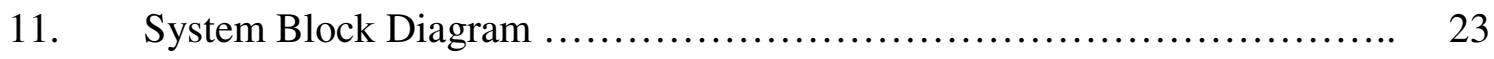

12. Cross-Section across Short Axis of MEb ........................... 28 


\section{Chapter 1}

\section{Introduction}

Medical devices provide treatments and therapies for many diseases or disabilities, such as pacemaker preventing a heart attack, to a prosthetic leg allowing someone to engage in physical activity. However, a recent area of interest includes pain management therapy. Approximately 10 to $12 \%$ of the population is affected by the common disease of a migraine. The World Health Organization has labeled this common disease as one of the most disabling neurological disorders [1].

Advanced Bionics is a medical device company developing a neural stimulation device to help treat migraines and chronic headaches. A thesis group consisting of four biomedical engineering graduate students worked on testing the device and developing future tests for their device. One of the hazards for this device is the amount of heat dissipated during the recharging of the stimulator's battery. The battery is inductively charged by an electromagnetic field and thus heat is generated within the device. The device cannot exceed a certain temperature change per the Food and Drug Administration guidance regulations. This thesis discusses the tests that can be performed to test the heating of the device, the testing of an internal current and voltage, the ergonomics of the device, statistical analysis of data, and the development of a model head simulating an in-vivo environment. 


\section{Chapter 2}

\section{Device Applications}

\subsection{Migraines, Headaches, and Pain Treatment}

Everyday migraines and chronic headaches affect millions of people. Current medications and treatments cannot alleviate the pain associated with migraine or headache for some people. The neural stimulation device created by Advanced Bionics gives hope to these patients.

Migraines can occur at varying intervals that can last from 4 to 72 hours. During a migraine, a throbbing pain will typically occur in the frontal parts of the cranium and sometimes it is intense enough to disrupt daily activities. Nausea, vomiting, and light phobia arise from the intense pain [1].

Cluster headaches are rare, extremely painful and debilitating headaches that occur in clusters. Patients endure excruciating pain that is described as severe headaches of a piercing quality near one eye or temple that last for fifteen minutes to three hours, some lasting days [2].

Pain is treated in many ways depending on the cause and severity of pain, such as physical therapy, strong medication, and surgery. Pain is a very subjective experience since the amount of pain perceived varies based on the intensity of the signal and the transportation to the brain. Chronic pain is very complex and difficult to diagnose, but can be treated by an electrical stimulation used to help alleviate the pain and help people 
return to a higher quality of life. Pain is an electrical signal that travels along nerve fibers to the brain. Delivering doses of electricity to the nerve fiber can help mask these pain signals. The pain signals are interpreted by the brain as a pleasant sensation called paresthesia. Most people describe the feeling of paresthesia as a gentle tingling, warm sensation, or massage [3].

\subsection{The Multi-Electrode bion}

This thesis explores the testing, use, and future projects involved with an implantable device used to treat chronic headaches, intractable headaches, intractable migraines, and cluster headaches called the Multi-Electrode bion (MEb). The MEb is currently being developed by Advanced Bionics, a Boston Scientific Company and an example of the device's appearance is shown in Figure 1.

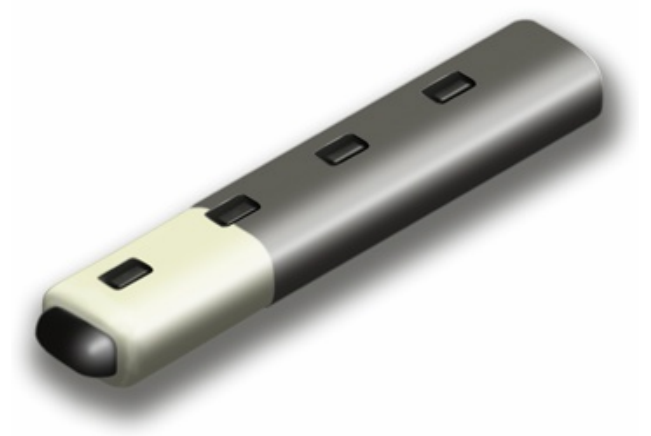

Figure 1. Illustration of possible Multi-Electrode bion (MEb)

The device treats the pain caused by these headaches via stimulation to the patient's greater occipital nerve. The MEb main components consist of stimulating electrodes, telemetry, and a rechargeable battery. The dimensions of the MEb measure $29 \mathrm{~mm}$ long by $8 \mathrm{~mm}$ wide by $5 \mathrm{~mm}$ thick, with an oval shape to all of its edges. It is designed to be 
leadless and lasts about one day for the average patient before requiring recharging. Implantation occurs subcutaneously into the neck area above the spine and below the base of the skull. This is approximately at the first cranial nerve (C1), as shown in Figure 2. The implant depth form the surface of the skin to the shallowest surface of the implant is recommended to be no less than $0.3 \mathrm{~cm}$ and no greater than $1.3 \mathrm{~cm}$.

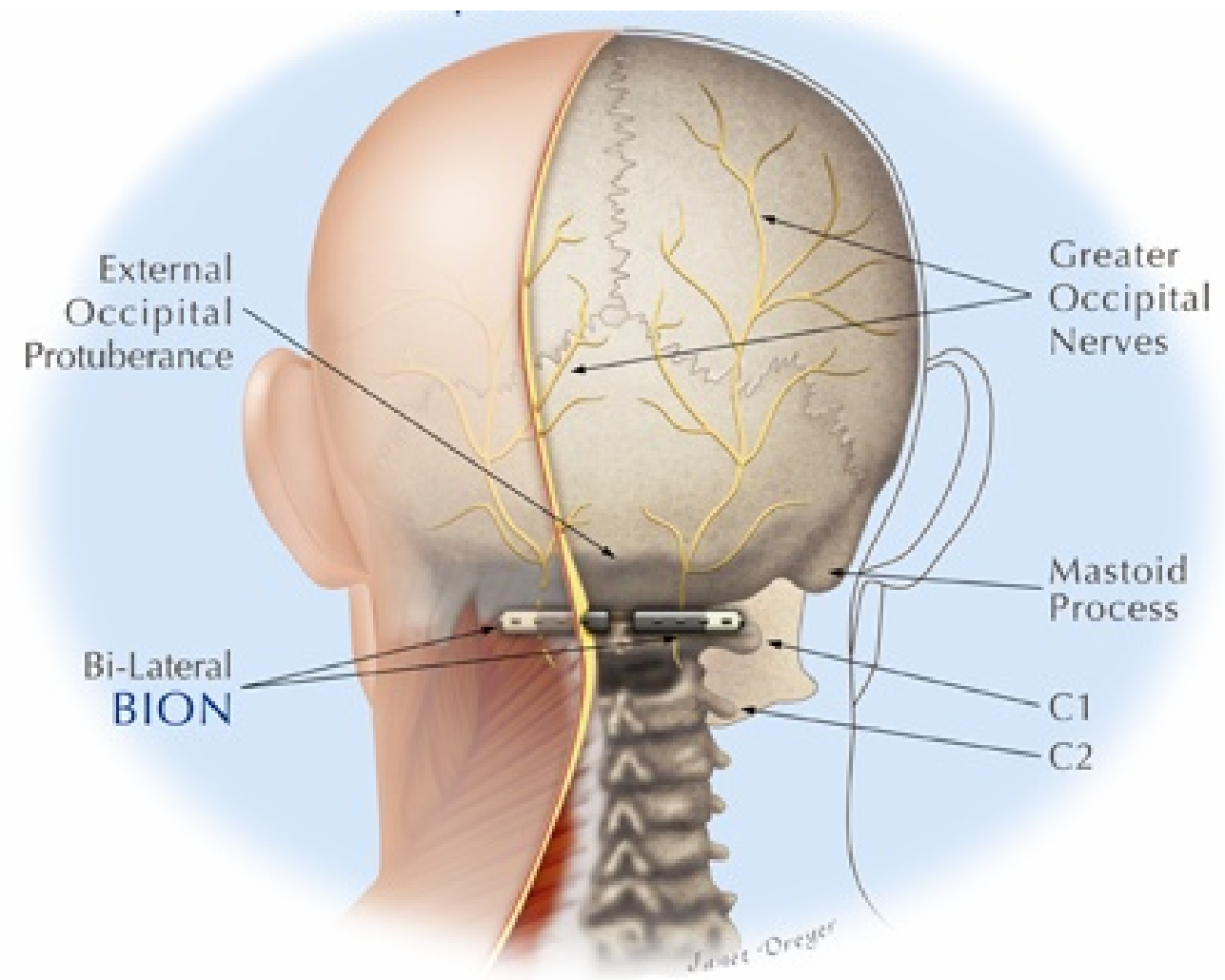

Figure 2. Illustration of the implantation

\subsection{Mobile Charging System}

The entire system consists of one or two MEbs, a mobile charging system, surgical tools, and a remote control. The MEb mobile charging system portability allows patients to continue performing daily activities while recharging their MEb. The charging system 
creates a magnetic field to transfer energy that recharges the device. It also provides forward and reverse telemetry for communication to and from the device, and contains temperature sensors to monitor the surface temperature of each device. The mobile charging system transcutaneously recharges the battery of one or two implanted MEbs by inductive coupling. It takes approximately two hours in order to achieve a 95 percent capacity of the battery when using the charging system. The mobile charging system allows the patient to perform some light physical activity, such as sitting, walking, and lying down supine, without charge interference.

The system consists of the charger module, coil module, and alternating current (AC) adapter. The charger module size is comparable to a cell phone and can be worn with a belt clip or pouch. The coil module will be held in place with the use of a coil positioner such as an elastic headband as shown in Figure 3. The charger is portable and worn at the back of the neck. The ergonomic design aligns with the bottom of the head and upper portion of the neck to achieve optimal coupling with the implant. The charging system is designed to be comfortable, aesthetically pleasing, and efficient at charging. It also avoids interference with household equipment when charging, such as a television, cell phone, radio, etc. 


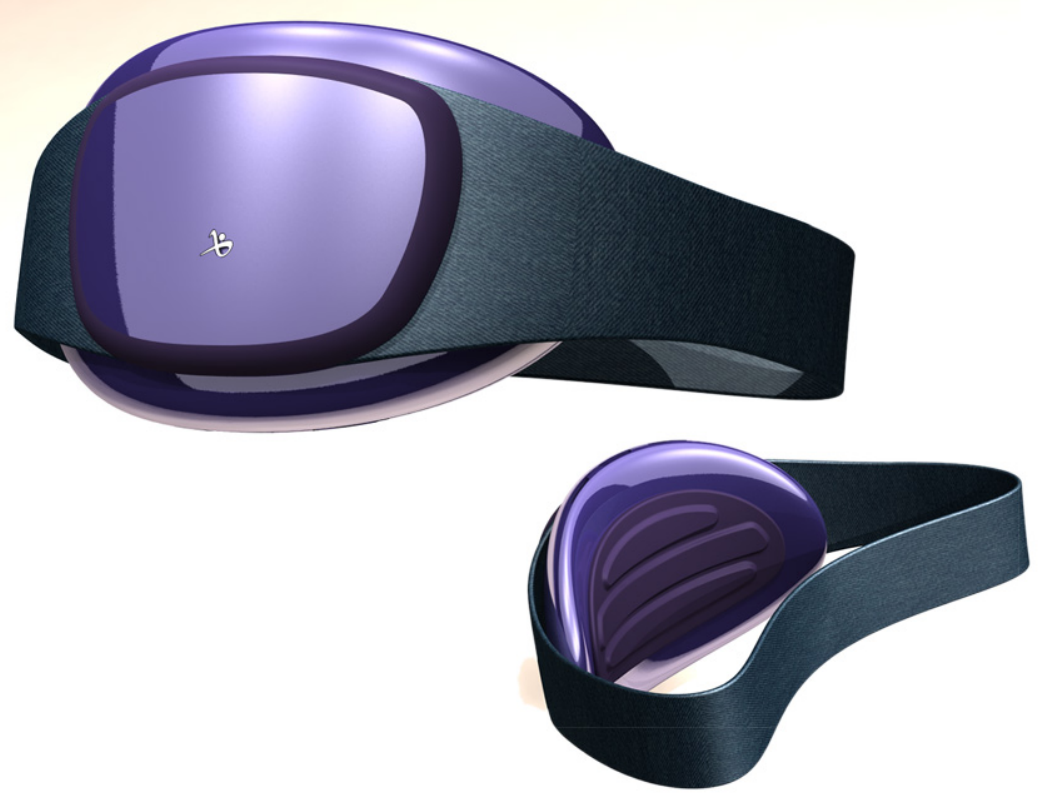

Figure 3. The mobile charge system's coil positioner

The mobile charging system also provides forward and reverse telemetry for communication to and from the MEb. Telemetry is transmitted and received through the same coil that is used for charging and allows information to be recorded or commands to be sent to the device.

The charging system will inhibit charging if the temperature of the device exceeds critical levels, at which the device or patient's tissue can become damaged or cause discomfort. The remote control shows the battery status on the implant(s) and allows patients to increase or decrease the amplitude of impulses delivered or stop stimulations. This allows the patient to enhance their treatment when experiencing an episode of pain and 
continuously be informed on the device's battery status. An example the appearance of a remote control is shown in Figure 4.

The clinician will set a range of acceptable amplitudes that are appropriate for the patient's level of pain.

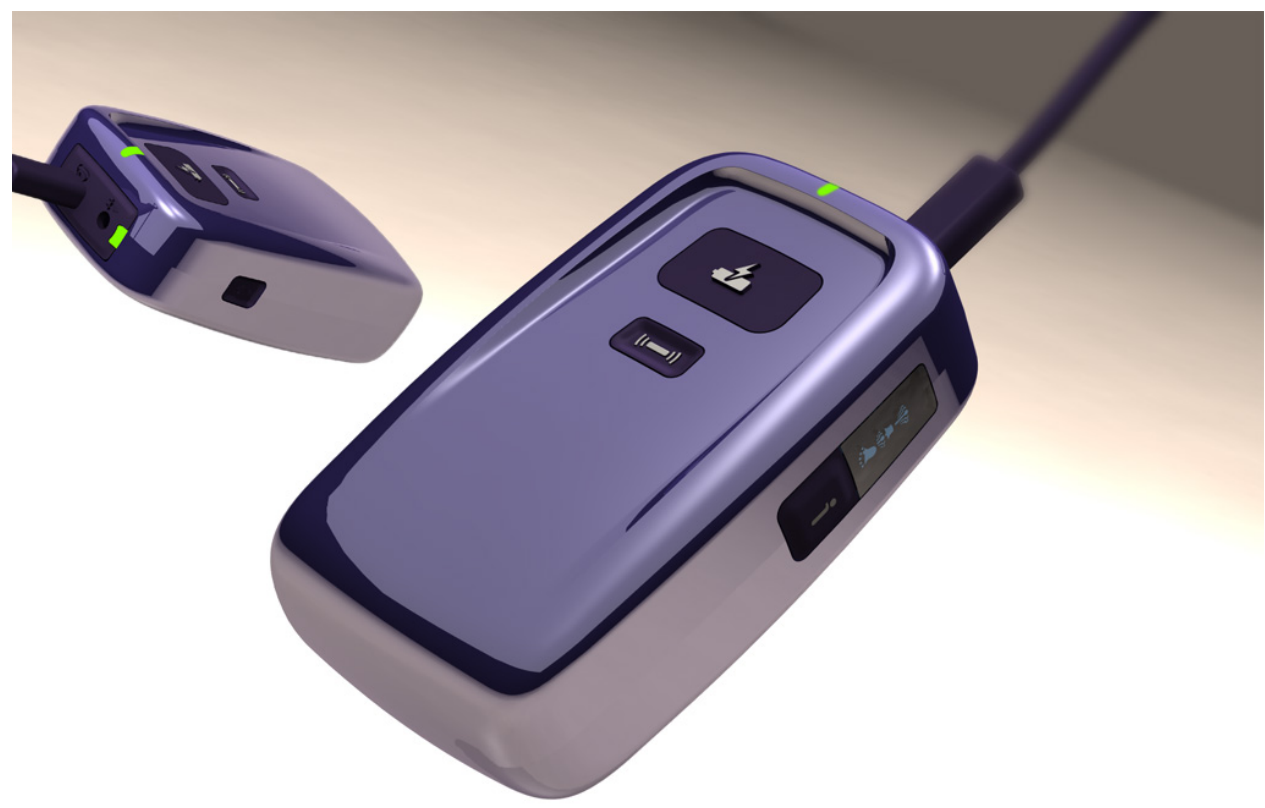

Figure 4. Illustration of possible Remote Control

The amplitude of the current ranges from $0.1 \mathrm{~mA}$ to $24.8 \mathrm{~mA}$ amplitude, with a step size no greater than $0.1 \mathrm{~mA}$, when the amplitude is less than $12.0 \mathrm{~mA}$ and with step size no greater than $0.2 \mathrm{~mA}$ when the amplitude is greater than or equal to $12.0 \mathrm{~mA}$. The importance of step sizes is to gradually increase the current so that the person does not have an adverse effect to a large stimulation. Biphasic pulses are used by the electrodes to cause stimulation. Biphasic amplitudes are great for maintaining the battery life while 
still providing a sufficient stimulation. There is also an available burst mode, which cyclically activates stimulation for the on time and deactivates for the off time. Another option available is the soft start where stimulation is linearly ramped from $0 \mathrm{~mA}$ to the value set in the amplitude register. The typical stimulation parameter is $60 \mathrm{~Hz}, 250$ microseconds, and $8 \mathrm{~mA}$. The battery should last 24 hours at these standard parameters. There will only be three electrodes on each side of the MEb providing stimulation. The device will sterilized via ethylene oxide and the casing of the MEb is biocompatible. 


\section{Chapter 3}

\section{The Nervous System}

The nervous system controls the communications throughout the human body. Cells communicate via electrical and chemical signals, which are very fast, specific, and elicit a response. The nervous system is divided into two parts, the central and peripheral nervous system, CNS and PNS respectively. The brain and spinal cord make up the central nervous system and are responsible for interpreting signals and dictating a response. The peripheral nervous system consists of all nerves, extending from the brain and spinal cord, outside of the CNS. Nerve cells, or neurons, are the basic structural units of the nervous system. Some extraordinary neuron characteristics include an extreme longevity, they cannot reproduce, and they have a high metabolic rate requiring continuous oxygen and glucose.

\subsection{The Neuron Structure}

There are four main components of a neuron, the cell body, dendrites, axon, and axon terminal. The cell body, which is considered the center of the neuron, contains a nucleolus and all of the usual organelles found in cells (i.e. mitochondria, vacuoles, etc.). Branchlike processes that extend from the cell body are dendrites and axons. Dendrites branch directly from the cell body and correspond to the main receptive regions. They direct incoming signals toward the cell body. Each neuron has a single axon protruding from the cell body. The cell body and axon attach at the axon hillock, which is the coneshaped area of the cell body and then the axon forms into a slender process that is 
uniform in length. The axon then branches profusely at its end into the axonal terminal. The axons generate nerve impulses and transmit them away from the cell body. When the impulse reaches the axonal terminal, it causes chemicals, or neurotransmitters, stored in vesicles to be released into an extracellular space which will excite or inhibit a nearby neuron. An illustration of a neuron is shown in Figure 5.

Many nerve fibers, especially longer and large diameter fibers are covered in a myelin sheath. Myelin, is a whitish, fatty protein lipoid that protects and helps electrically insulate fibers from one another while also increasing the speed of nerve impulse transmissions. The difference in transmission speed from myelinated to unmyelinated fibers can be on the order of 150 times faster in myelinated fibers. There are gaps in the sheath called nodes of Ranvier which occur at regular intervals, about $1 \mathrm{~mm}$, along the myelinated axon. These nodes are the areas in which action potentials occur. 


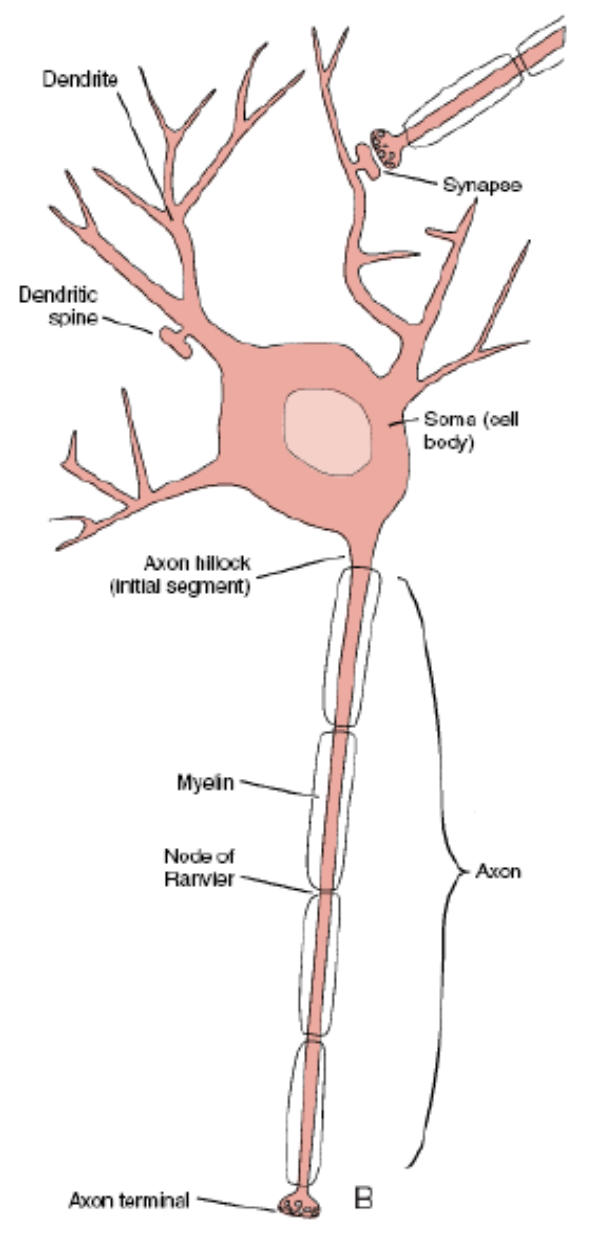

Figure 5. The Basic Structure of a Neuron

\subsection{Action Potentials}

An action potential, or nerve impulse, is always the same, regardless of the source or type of stimulus and it is the foundation for all functional activities in the nervous system. Overall the human body is electrically neutral since it has the same number of positive and negative charges. However, some areas have a dominating charge making the region either positively or negatively charged. Work is done to separate these charges such as charges separated in a battery. 


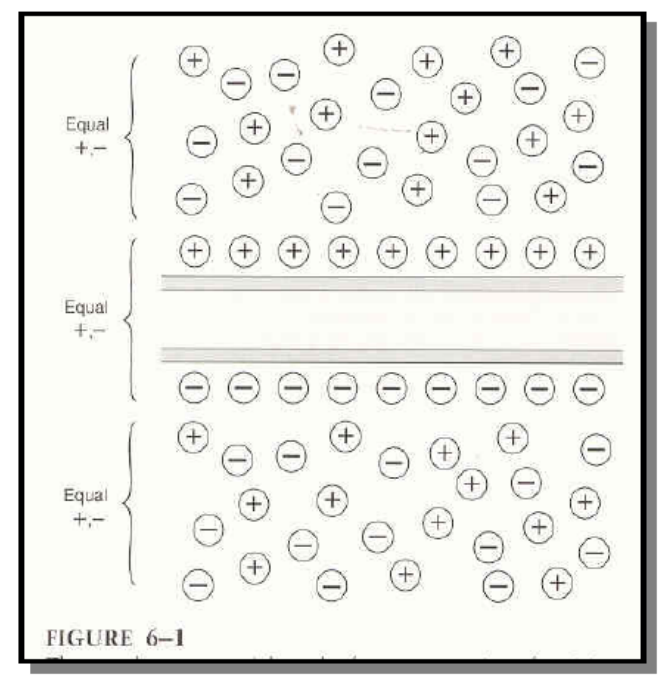

Figure 6. The Charge Separation of Ions on a Cell Membrane

In order to understand an action potential, voltage, current, and resistance must be defined. Voltage is the measure of potential energy generated by these separated charges. The voltage measured between two points is called the potential difference. The greater the difference between charges at the two points, the higher the voltage. Current is the flow of electrical charges from one point to another and can be used to perform work. Resistance is the hindrance to charge flow. The relationship between voltage, current, and resistance is then given by Ohm's law: $V=I^{*} R$, where $V$ is voltage, $\mathrm{I}$ is current, and $\mathrm{r}$ is resistance.

Current is directly proportional to the voltage, or in other words, the greater the voltage, the greater the current. In vivo, electrical currents occur due to the flow of ions across cellular membranes. There is a slight difference in the numbers of positive and negative ion on the two sides of cellular plasma membranes, thus there is a voltage potential. There are ion channels made up of membrane proteins embedded along the plasma 
membranes. There are many different types of ion channels, such as passive, active, and gated channels. Gated channels have a protein molecule that can change shape in response to various signals. Chemically gated channels open when the appropriate neurotransmitter binds. Voltage gated channels open and close in response to changes in the membrane potential shown in Figure 7. Each type of channel is selective to the type of ion(s) it allows to pass. When gated ion channels are open, ions diffuse quickly across the membrane following their electrochemical gradient. Ions passively move along chemical gradients of areas of high concentration to areas of low concentration, while ions moving along an electrical gradient move toward an area of opposite charge. Combined the electrical and chemical gradients form the electrochemical gradient.

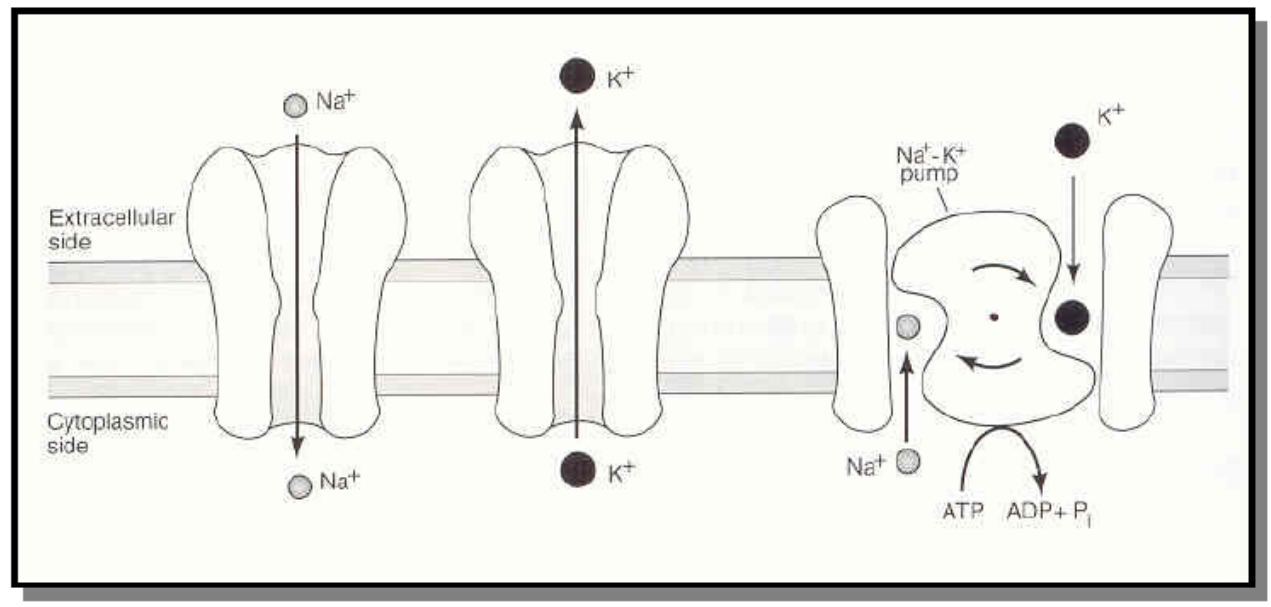

Figure 7. Voltage Gated Ion Channels and an ATP Sodium-Potassium pump, respectively 
The potential difference between two points is measured by using two microelectrodes connected to a voltmeter. One microelectrode is placed inside neuron while the other rests on its outside surface shown in Figure 8. The voltage of a resting neuron, called the resting membrane potential, varies from $-40 \mathrm{mV}$ to $-90 \mathrm{mV}$ in different types of neurons. The negative sign of the potential indicates that the inside of a neuron is more negatively charged with respect to the outside. This is because there are more potassium ions $(\mathrm{K}+)$ inside a neuron and more sodium ions $(\mathrm{Na}+)$ on the outside of the cell. Potassium and sodium are both positively charged ions, but sodium has a larger positive charge associated with it than potassium. There are other ions inside and outside the cell but sodium and potassium play the most important role in generating the membrane potential. Potassium ions diffuse out of the cell along their concentration gradient much more readily and quicker than sodium ion can enter the cell. However, in order to maintain the higher concentration of sodium outside of the cell and potassium inside, the cell uses a protein channel that requires energy. ATP-driven sodium-potassium pumps remove three sodium ions from inside the cell and then transports two potassium ion back into the cell in order to stabilize the resting membrane potential. ATP, adenosine triphosphate is a nucleotide that is the standard energy unit consumed in the body. With the energy provided by the ATP, the sodium-potassium pump can perform work to move the ions against their concentration gradients. 


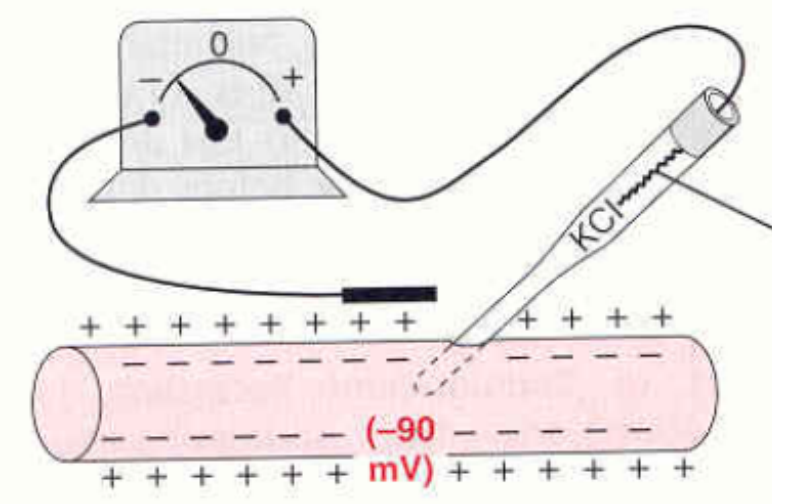

Figure 8. A Voltmeter reading the Membrane Potential of a Neuron

The primary way neurons communicate is by generating and propagating action potentials. An action potential is a brief reversal of membrane potential with a change of voltage over a few milliseconds. A neuron transmits a nerve impulse only when it is adequately stimulated. The stimulation changes the permeability of the neuron's membrane by opening specific voltage-gated channels that are located on axons. Only axons are capable of generating action potentials. These channels open and close in response to changes in the membrane potential and are activated by local currents that spread toward the axon along the dendritic and cell body membranes. Depolarization is a reduction in the membrane potential, or the inside of the membrane because less negative than the resting potential.

There are four main stages in an action potential, the resting state, depolarization phase, repolarization phase, and hyperpolariztion phase as seen in Figure 9. At resting state, the voltage gated channels are closed, however there is a small amount of leakage of ions across the membrane. The activation gate is closed while the inaction gate of the voltage 
gated channel is open. Both gates must be open for the channel to allow sodium ions to enter. A potassium channel has a single voltage sensitive gate that opens slowly during depolarization.

During the depolarization phase, there is an increase in sodium permeability and the membrane potential increases to a less negative voltage. The local currents cause the sodium channel activation gates to open quickly and sodium rushes into the cell. When depolarization of the membrane reaches a certain critical level called threshold, then depolarization becomes a self-propagating by positive feedback. Threshold levels are often between -55 and $-50 \mathrm{mV}$. During depolarization, sodium ion permeability is about 1000 times greater than it is in a resting neuron. Consequently, the membrane potential becomes less negative and overshoots to about $+30 \mathrm{mV}$ as sodium ions rush in along their electrochemical gradient. The depolarization phase is seen as the rising of the "spike" of an action potential.

During the repolariztion phase, there is a decrease in sodium permeability. The rising phase of the action potential lasts for about $1 \mathrm{~ms}$ and is limited by increasingly positive membrane potential to more positive ions entering. Also, the slow inactivation of the sodium channels have reacted and close. As a result the action potential spike stops and reverses direction. There is an increase in potassium permeability because the slow voltage sensitive potassium gates open and potassium rushes out of the cell following its electrochemical gradient. Thus the internal negativity of the resting neuron is restored. 
The hyperpolarization phase occurs because potassium permeability continues due to the slow nature of potassium gates. The hyperpolarization, also called the undershoot, is seen on the recording as a slight dip following the action potential spike. Hyperpolariztion occurs when the membrane potential or voltage increases, becoming more negative than the resting potential. After the action potential, the sodium-potassium pump redistributes ions to its original ionic distribution [2].

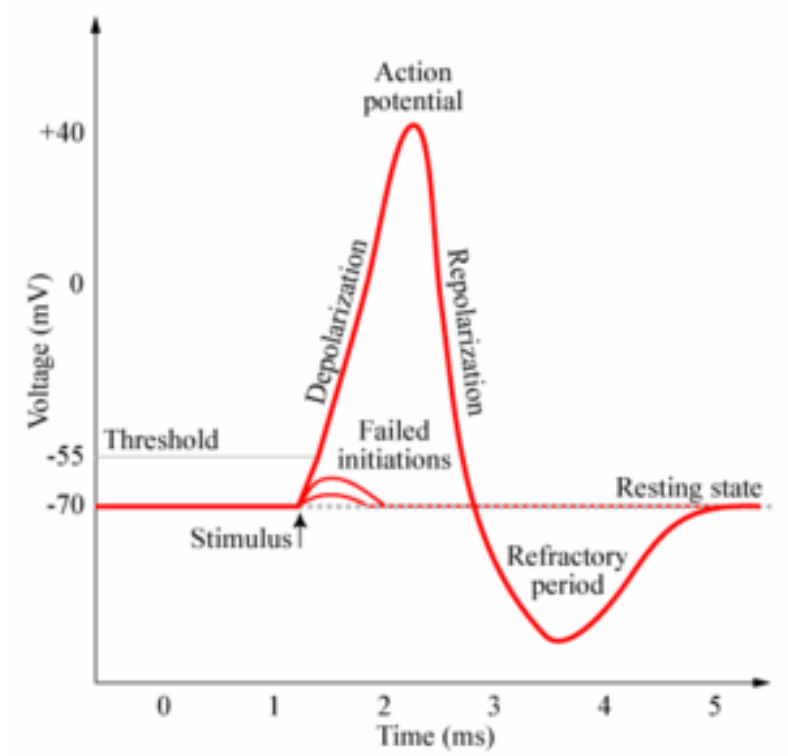

Figure 9. The stages of an Action Potential

The action potential must be propagated along the axon's entire length in order to transmit a signal. The influx of positive ions along the membrane causes a local current flow that causes depolarization of adjacent membrane areas in the distal direction, away from the origin of the nerve impulse. Action potentials are initiated at one end of the axon and conducted away from that point toward the axon's terminals. Once initiated, an action potential will self propagate at the same velocity down the entire length of the axon. An action potential is an all-or-none phenomenon, in the sense that it either 
happens completely or it does not happen at all. In order for an action potential to completely occur, it first needs to reach the threshold voltage at which the membrane will depolarize. In order for a stimulus to cause an action potential, the frequency and amplitude of the electrical stimulus must be great enough to reach the threshold voltage. However, over-stimulation of a nerve, due to high frequencies and amplitudes, will result in irreversible nerve damage. There is a period after a stimulation called the absolute refractory period, in which the nerve fiber cannot be stimulated again due to the gates of the sodium channels being closed. This ensures that the action potential is an all or nothing response and that there is a one-way transmission of the signal.

Conduction velocities of axons vary with the size of the axon diameter and influence of a myelin sheath. Nerve fibers that conduct impulses quickly, at $100 \mathrm{~m} / \mathrm{s}$ or faster, are found in neural pathways where speed is essential. As a general rule the larger the axons diameter, the faster the nerve conduction velocity. This is due to the fact that there is more resistance to the flow of local current, and thus surrounding areas of the membrane can depolarize more quickly and be brought to threshold. Action potentials travel much faster in myelinated fibers via salutatory conduction than in a continuous conduction. Continuous conduction occurs in unmylenated axons, and action potentials are generated at the sites immediately adjacent to each other and the conduction of the signal is relatively slow. The presence of a myelin sheath dramatically increases the rate that the impulse travels along the axon. This is because the myelin sheath acts as an insulator and prevents the leakage of charge from the axon. There are areas along the myelin sheath 
where an action potential is regenerated called the nodes of Ranvier. The electrical signal jumps from node to node along the axon, thus the conduction velocity is much faster.

A synapse is a junction that mediates information transfer from one neuron to another neuron or effector cell. The neuron conducting impulses toward the synapse is called the presynaptic neuron and the neuron that transmits the electrical signal away from the synapse is called the postsynaptic neuron. The presynaptic neuron is the information sender, and the postsynaptic neuron is the information receiver. There are electrical and chemical synapses used to transmit information. Chemical synapses are specialized for release and reception of chemical neurotransmitters. An axonal terminal contains many tiny, membrane-bounded sacs called synaptic vesicles which contain thousand of neurotransmitter molecules. There is a receptor region on the membrane of a dendrite or the cell body of the postsynaptic neuron which has several neurotransmitter receptors. The presynaptic and postsynaptic membranes are very close but separated by the synaptic cleft, which is a fluid-filled space that is about 30 to $50 \mathrm{~nm}$ wide.

Transmission of signals across the synapse is chemical and depends on the release, diffusion, and receptor binding of neurotransmitter molecules and causes a unidirectional communication between neurons. Signals travel along an axon as an electrical signal, then is converted to a chemical signal at the synapse, then back to an electrical signal in the next neuron. When a nerve impulse reaches the axon terminal, it sets into motion a chain of events that triggers neurotransmitter release. The neurotransmitter crosses the 
synaptic cleft and binds to receptors on the postsynaptic membrane which causes changes in the postsynaptic membrane permeability [4].

When the nerve impulse reaches the axonal terminal, depolarization of the membrane opens not only sodium channels but voltage-gated calcium channels. Calcium floods into the terminal from the extracellular fluid and causes the synaptic vesicles to fuse with the axonal membrane and empty their contents by exocytosis into the synaptic cleft. The calcium is then used up by the cell or removed by a pump. The neurotransmitter binds to a postsynaptic receptor by diffusing across the cleft as seen in Figure 10. As the receptor proteins bind neurotransmitter molecules, they allow ion channels to open in the postsynaptic membrane. As the ions flows, it causes a depolarization in the membrane potential. The higher the frequency of impulses that reach the terminal, or the more intense the stimulus, the greater number of synaptic vesicles will fuse and spill their contents and the greater the effect on the postsynaptic cell. The effects of neurotransmitters last a few milliseconds before being degraded by enzymes, removal from the synapse back to the neuron, or diffusion of the neurotransmitter away from the synapse. 


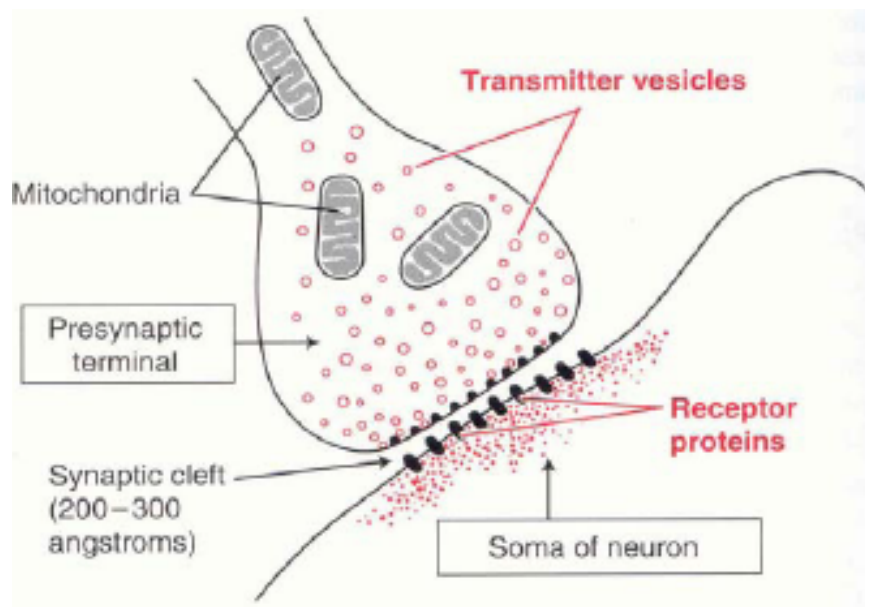

Figure 10. Synaptic Cleft and Neurotransmitters

There are two types of synapses, excitatory postsynaptic potentials and inhibitory post synaptic potentials. At excitatory synapses, neurotransmitters bind and cause depolarization. Binding of neurotransmitters at inhibitory synapses reduces a postsynaptic neuron's ability to generate an action potential. A single EPSP cannot induce an action potential in the postsynaptic neuron, but many EPSPs can cause threshold depolarization. Most neurons receive both stimulatory and inhibitory inputs from thousands of other neurons. Both of these signals summate and if the EPSPs dominate the membrane potential enough, the membrane will reach threshold and fire [4].

The combination of the anatomy of the neuron, ion concentrations, membrane potentials, action potential stages, neurotransmitters, and receptors send information throughout the body. 


\section{Chapter 4}

\section{Testing Methods}

The FDA requires tests to be performed before granting approval for a device's release in the United States. Our thesis group thought of numerous testing methods that could be performed to identify the problematic areas of the device before or during animal testing. Some methods of testing the heat dissipated by the device included calorimeter, thermistor, and infrared testing. We also performed some analysis of a sample MEb to compare actual and theoretical values from the charging and discharging cycles.

\subsection{Lab View Testing}

Advanced Bionics provided our group with a sample MEb, external charging board, and program interface in LabView. This allowed us to test different MEb charging and discharging parameters. Through LabView we communicated commands to the device via a USB cord that connected the external charging board, which was also connected to the portable charging system, to the computer. It provided a way to measure and automate collecting data from the physical world and storing it, while also sending commands to change parameters. A block Diagram of the LabView test set-up is shown in Figure 11. 


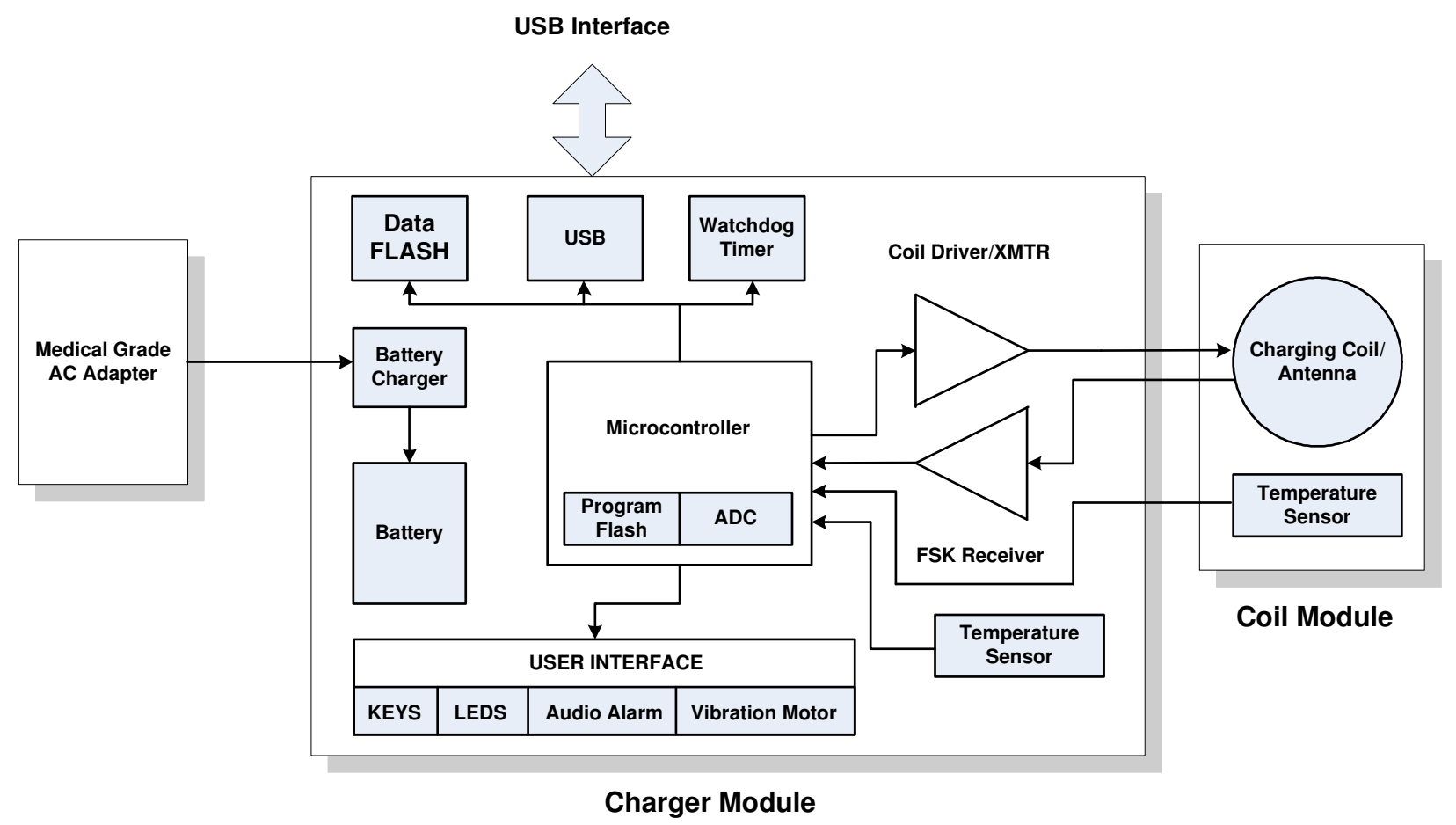

Figure 11. System Block Diagram

The purpose of the LabView testing was to compare actual and theoretical charging rates at different parameters. The device required a power source that was limited to two amps. Then the USB cable connected the external charging board and the computer. Next, the LabView program was initiated and synchronized with the MEb. The program commands operated reverse hexadecimal, which means all voltages we desired to test needed to be converted to hexadecimal and then reversed. An oscilloscope was connected by current probes to the device ensuring an electric field was present and being emitted from the charging coil by attaching. The sample MEb was checked and found to be functional and responding to the program commands. Then we set and checked the values of battery current registry setting, charging coil current, device coil current, battery voltage, and voltage of a specific node inside the circuitry call $\mathrm{V}_{\text {nab. }}$. A one kilo ohm resistor was 
attached to the MEb to verify stimulation control functioned properly. We then began recording the quantitative data required for parameter characterization.

\subsection{Calorimeter, Infrared, Thermistor Testing}

An easy and efficient test of the heating of a device is through a calorimeter. Calorimetry is the science of measuring the heat of chemical reaction, physical changes and heat capacity. A calorimeter is device used to measure the heat generated by reaction by measuring changes in volume, resistance, or distribution of light. Since this device emits heat during the recharge cycle as a result of the internal electronic circuitry, the focus of this test should be the temperature. Testing the device in different orientations and in different mediums, such as air or agar, will correlate the effects of these variables [5].

Infrared (IR) cameras show where heat is generated on the surface of objects. This method of testing would be very beneficial for the MEb since it identifies which areas generate the heat the most, fastest, and retain it. The exact temperature cannot be measured by the IR camera, but it can approximate which areas are the hottest compared to the overall device. Since heat is caused by the motion of molecules, IR cameras detect an electromagnetic radiation emitted from random movement of particles in an object. The surface temperature is a function of the amount of radiation emitted from the object which is then emitted as a certain wavelength by the IR camera [6]. This has a linear relationship of increasing the temperature causing an increase in the wavelength of the IR image. IR cameras are very accurate and easy to use, which is necessary in the repeatability of testing the $\mathrm{MEb}$ device at different parameters. 
Thermistor testing would provide accurate data and also a way to store the information. A thermistor is a type of resistor used to measure temperature changes. A large change in resistance is proportional to a small change in temperature. Thermistors are one of the most accurate types of temperature sensors with an accuracy of 0.1 or 0.2 degrees Celsius. The MEb temperature change is relatively small, only about a 5 degree Celsius change. Thermistor testing is most useful when used with IR testing, because the hot spots that are determined using IR cameras can be quantified with the placement of a thermistor. Thermistor testing can be performed in different orientations, directly, or through a medium to determine the effects on the MEb [7].

\subsection{Battery Heating}

Since the device is very small, the heating of the battery has a large effect on the MEb, which is in direct contact with the subcutaneous fat layer. The battery easily transfers the heat that it produces to the surrounding tissue during recharging. If a surface is bounded by a conductor such as the metal casing of the $\mathrm{MEb}$, then a changing magnetic field will induce a voltage around that conductor causing a current to flow, thus generating heat [8]. Neurons cannot be exposed to temperature changes above 4.5 degrees Celsius because the proteins embedded in the membrane begin to denature. Therefore the MEb should not exceed a 2 degree Celsius temperature change during normal operation and a 4 degree Celsius change during charging. Understanding the thermal characteristics of the battery during charging and discharging is important for patient safety and battery longevity. The goal is to find the correct balance between the charging rate and temperature change 
of the MEb. The faster the device is recharged, the greater the temperature dissipated. However, in order to keep the patients happy, a slow charging rate could appear tedious and uncomfortable to wear the charging system headset for many hours at a time.

\subsection{FDA Approval}

The United States Food and Drug Administration is responsible for regulating medical devices that are sold in the United States. The FDA works closely with companies before their medical device products are released by listing a set of standards and requirements that they deem necessary for approval. This includes providing detailed documentation of the manufacturing process, production, design specifications, and results from animal testing. The FDA will then come to the companies and performs inspection and then evaluates all of the documentation that was submitted in order to give approval for product release. Companies begin to work with FDA once they have decided that they would like to release a product. This process can be several years and requires detailed documentation and testing in order to be released in the United States.

Advanced Bionics' MEb will be implanted into the back of the neck of a human and will provide an electrical pulse to nerves. Advanced Bionics has started showing tests and documentation that the MEb is safe for human implantation by its biocompatibility, temperature changes, and electrical current levels. Animal testing was performed on dogs in order to simulate the in vitro conditions that are necessary to show that the device is safe for human implant. The FDA required that the Advanced Bionics MEb device would not exceed a four degree Celsius change during the recharging cycle of the device 
due to the heat that is generated in the battery of the device when it is inductively charged and dissipated to the surrounding tissues from the casing [9].

\subsection{Head Model}

The creation of a head model could help alleviate some of the tedious testing that the FDA requires and would help increase the repeatability, time consumption, accuracy, and efficiency of testing for the MEb system. Since preliminary testing in humans is unsafe and prohibited, validation testing is necessary to determine how the device will perform in vivo. After performing some preliminary testing such as infrared, calorimetry, and thermistor testing, the device will be tested in-vitro usually through an animal. Animals with similar environments to humans is the most common form of testing to validate the data results assuring the device is safe for humans. If a head model was created and approved by the FDA as a valid test, this would help reduce the amount of animal tests. Animal testing is expensive, time consuming, and usually outsourced with another company. This would ultimately help save time and money that is often necessary for validation. The model would need to simulate the environment inside of the head. This includes tissue conductivity, geometry, and temperature.

The elements that should be modeled include the skull, neck, scalp, hair, muscle, fluids, blood, skin, brain. Using the visible human project data, one can easily obtain dimensions of the human body with excellent resolution. The data from the visible human project is given in two forms, CT scans and MRIs [10]. This will provide the correct geometry and dimension for creating a human head model of the bone and tissue. Bone can be 
simulated by using a real bone, hydroxyapetite, or through rapid prototyping of a ceramic with similar density. The simulated tissue should be similar in thickness and consistency. An agar gel is the easiest medium that could be applied to this model. Currently agar gel is used to simulate the environment of tissue for some tests. A polymer, like latex could replicate the taut nature of the skin. Some other important environmental factors that would need to be simulated include the $\mathrm{pH}$, salinity, temperature, and conductivity [11]. These factors could be simulated by a saline suspension that flows throughout the head and the fluid could be heated to 36.2 degrees Celsius or placed in a hood set at this temperature.

For the purpose of this test, the most important element to simulate is the fatty subcutaneous layer that the device will be implanted into as seen in Figure 12. Creating the correct geometry, size, and environment are the most important elements.

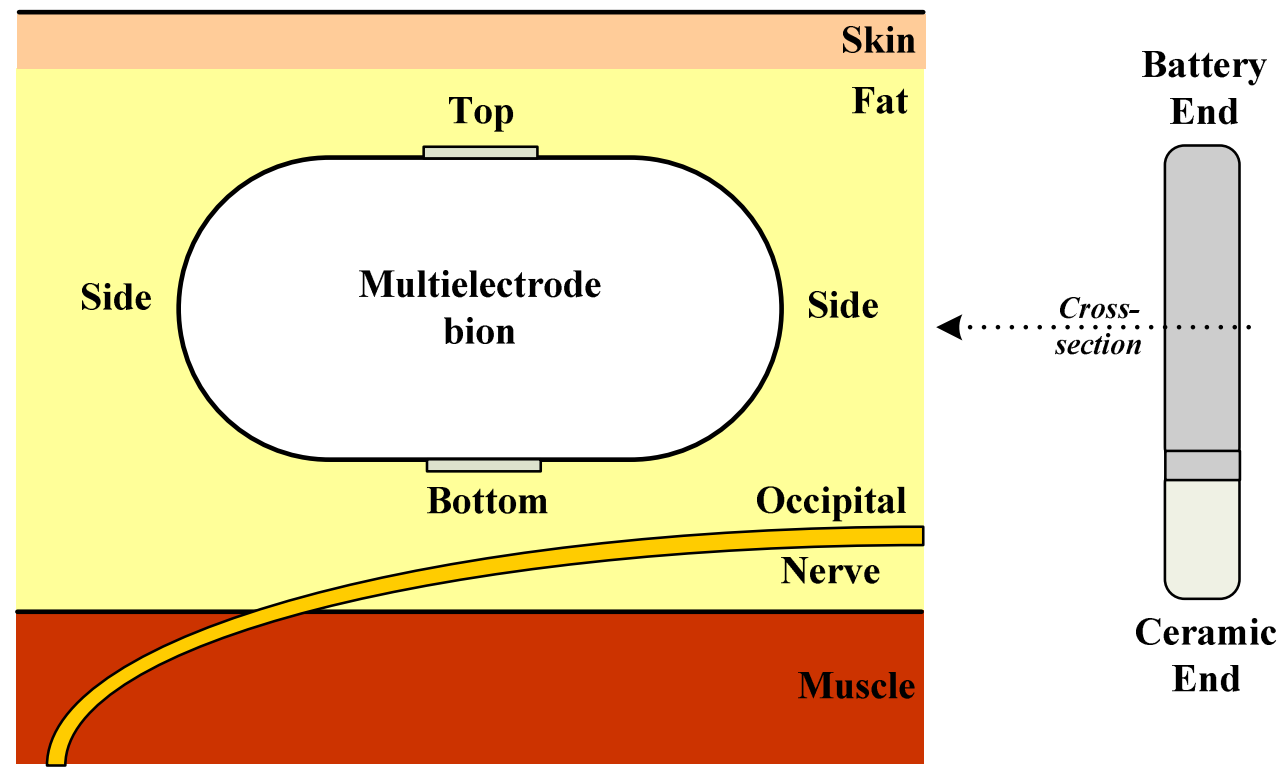

Figure 12. Cross-Section across Short Axis of MEb 


\subsection{Ergonomics}

The external charger and remote control are the only devices that the patient will have to interface with their MEb. Therefore it is important to make sure these components are ergonomic and easy to use. Testing can be performed to see the effects of different orientations, physical activities, and ease of use of the remote to make the whole MEb system more efficient.

If a medium was formed that could simulate the internal environment, then one could perform testing to see if there is a difference in the efficiency of the recharging cycle by performing various activities such as walking, running, driving, cleaning, sitting, standing, lying down, etc. One could attach the simulated medium onto the back of their neck and perform various physical activities while wearing the mobile external charger. Other factors that could change the recharging efficiency could be from long hair versus short hair. Testing could also be performed to see the different charging rates with different orientation or placement of the external charger. Some patients may wear their charging device incorrectly and it would be beneficial to know the effects of this on the charging rate. A test could be performed by wearing the external charger in different orientations. Human tissue varies greatly person to person, so the tissue thickness would also vary and tests should be performed to evaluate the effects.

Another important factor that the external charger will need to consider is patient comfort and care. Performing tests on the comfort of the external charger will ensure that that the patient will have a more pleasant experience with the entire process and will help the 
charging rate. Since the device needs to be charged every day or every other day, the patient will want something that is not very noticeable and easy to use. This includes if padding were added to the external charger to gain some comfort, but knowing the effects it would have on the charging rate. An evaluation of the remote control LED buttons can be performed. Patients can be confused if there are too many buttons and options. Since the information provided by the remote control is very important, the remote control user interface should be analyzed [12].

\subsection{Design Considerations}

The design of any medical device that will be implanted needs to be designed with the patient's safety in mind. One of the most important characteristics of an implanted device is its biocompatibility. The device must not harm the host and the host must not harm the device. A biocompatible metal and ceramic casing was used to interface with the tissue so that the implant would evoke a minimal adverse response. An important thing to consider when approximating the depth of the implant into the tissue is that a fibrous capsule will surround the implant and cause slight impedance to the electrode stimulations.

The geometry of the device must be comfortable while implanted and easy for manufacturability. The oval shape of the MEb satisfies both of these conditions because round edges in vivo will cause less irritation, and the manufacturing of the device is easier since sharp corners are not efficient. If the device was larger, then the battery longevity would increase, however the re-charging time would also increase, and a larger 
field might be required in order to charge the battery to its full capacity. Also, the subcutaneous layer of the neck is very thin and a larger device might cause patient discomfort.

It could be suggested that the remote control easily be turned into a music player to increase the customer satisfaction with making the device less conspicuous. The concern with making the device have more than one function is that there is a greater possibility of failure. The reliability decreases when other features are added to a device. Another consideration is to charge the battery at the fastest rate possible. The problem with charging at a faster rate would cause more heating to be dissipated from the device. 


\section{Chapter 5}

\section{Conclusion}

Performing tests on these devices includes measuring the internal voltages and currents. A data crunching analysis of these devices can be performed by measuring the current at different register settings, and looking at the device inputs to make correlations with the output current. There is an equation which relates the battery's maximum current to the register setting by adding an offset current that is always present and multiply by a gain of unknown value. The gain and offset can be found by looking at the data results and performing certain analysis.

A statistical analysis can also be performed to calculate the average current and voltage, identify any outliers, and find the standard deviation which will show the consistency of the device's performance. Also further testing should be performed to see the effects of two MEb devices being charged at the same time. 


\section{BIBLIOGRAPHY}

1. Vincent, M., Hadjikhani, N., 2007. Headache Medicine in Brazil: Review The Cerebellum and Migraine. American Headache Society Journal Compilation, 820-825.

2. Schwedt, T., Dodick, D., Tretman, T., Zimmerman, R., 2005. Occiptial Nerve Stimulation for Chronic Cluster Headach and Hemicrania Continua: Pain Relief and Persistence of Autonomic Feature.

3. Hope for Chronic Pain Sufferers, retrieved June $3^{\text {rd }}$, 2007 from: http://www.controlyourpain.com/index.cfm?langid=1

4. Marieb, E. Anatomy \& Physiology. Benjamin Cummings, 2002. 334-368.

5. Cooke, L., Eliaszwi, M., Becker, W., 2007. Cutaneous Allodynia in Transformed Migraine Patients. Headache: The Journal of Head and Face Pain. 531-539.

6. Kennedy, C. Stancescu, M., Marriot, R., White, M., 2006. Recommendations for accurate heat capacity measurements using a Quantum Design physical property measurement system. Science Direct. 107-112. 
7. Jang, Y. 2003. A Contactless Electrical Energy Transmission System for PortableTelephone Battery Chargers. IEEE Vol. 5 No. 3, 530-538.

8. Wang, C., Covic, G. 2004. Power Transfer Capability and Bifurcation Phenomena of Loosely Coupled Indcutive Power Transfer Systems. IEEE Vol. 51 No. 1, 148-156.

9. Medical Devices, retrieved May $24^{\text {th }}$ from:

http://www.fda.gov/cdrh/index.html

10. Visible Human Project, retrieved June $15^{\text {th }}$ from:

http://www.nlm.nih.gov/research/visible/visible_human.html

11. Morgan, J., Sheridan, R., Burn Dressings and Skin Substitutes. Biomaterials Science. Elsevier Academic Press. 602-613.

12. Wickens, C., 2003. Introduction to Human Factors Engineering. New York: Addison Wesley Longman. 